\title{
Microstructure, Optical and Dielectric properties of Cerium oxide Thin films Prepared by Pulsed laser deposition \\ G. Balakrishnan ${ }^{*}$, Arun Kumar Panda ${ }^{2}$, Raghavan C.M. ${ }^{3}$, Akash Singh², M.N. Prabhakar ${ }^{4}$,
}

E. Mohandas ${ }^{2}$, P. Kuppusami ${ }^{5}$, Jung il Song ${ }^{4}$

${ }^{1}$ Department of Nanotechnology, Bharath Institute of Science and Technology, Bharath Institute of Higher Education and Research, Chennai-600073, India

${ }^{2}$ Materials Synthesis and Structural Characterization Division, Physical Metallurgy Group, Indira Gandhi Centre for Atomic Research, Kalpakkam-603102, India

${ }^{3}$ Aston Institute of Photonic Technologies (AIPT), Aston University, Birmingham, B4 7ET, UK ${ }^{4}$ Department of Mechanical Engineering, Changwon National University, Changwon, Republic of Korea

${ }^{5}$ Centre of Nanoscience and Nanotechnology, Sathyabama Institute of Science and Technology, Chennai600119, India

\begin{abstract}
Cerium oxide $\left(\mathrm{CeO}_{2}\right)$ thin films were deposited on $\mathrm{Pt}(111) / \mathrm{Ti} / \mathrm{SiO}_{2} / \mathrm{Si}(100)$ substrates using pulsed laser deposition (PLD) method at different temperatures such as, $300 \mathrm{~K}, 573 \mathrm{~K}$ and $873 \mathrm{~K}$ with $3 \times 10^{-2}$ mbar oxygen partial pressure. The prepared films were systematically investigated using X-ray diffraction (XRD), atomic force microscopy (AFM), photoluminescence (PL) and electrical measurement system. XRD analysis clearly shows improved crystallinity of $\mathrm{CeO}_{2}$ films prepared at 573 and $873 \mathrm{~K}$ substrate temperatures. The AFM analysis indicated the uniform distribution of the nanocrystallites and dense structure with the roughness (RMS) of $\sim 2.1-3.6 \mathrm{~nm}$. The photoluminescence (PL) studies of the films showed a broad peak at $\sim 366-368 \mathrm{~nm}$, indicating the optical bandgap of 3.37-3.38 eV. The electrical property study showed minimum leakage current density of $2.0 \times 10^{-7} \mathrm{~A} / \mathrm{cm}^{2}$ at 873 $\mathrm{K}$, which was measured at $100 \mathrm{kV}$ and this valuewas much lower than that of the $\mathrm{CeO}_{2}$ film deposited at $300 \mathrm{~K}$. The dielectric constants are increased and dielectric loss values decreased for the films with increasing substrate temperature.
\end{abstract}

Key words: X-Ray Diffraction, Thin films, Cerium oxide, Dielectric properties, Pulsed laser deposition, Electrical properties, Photoluminescence and Atomic force microscopy

*Corresponding author: balaphysics76@gmail.com ; (Dr. G. Balakrishnan) 


\section{Introduction}

High dielectric materials (high-k) are considered as a potential in the fabrication of electronic devices. There are different dielectric materials currently being used in metal oxide semiconductor field-effect transistors (MOSFETs), as gate oxide materials, as capacitor in memory devices and insulator in back-end interconnects [1,2]. The silicon dioxide $\left(\mathrm{SiO}_{2}\right)$ is highly compatible with silicon technology and exhibits high interface quality. However, direct tunneling of charge carriers occurs via the potential wall as the $\mathrm{SiO}_{2}$ layer thickness decreases, which in turn leads to increase in leakage current through gate dielectrics [3]. For thin $\mathrm{SiO}_{2}$ film, the leakage current from electron tunneling through the dielectrics is a problem [4].

There are series of important high- $k$ oxide materials, such as cerium oxide $\left(\mathrm{CeO}_{2}\right)$ [5,6], hafnium oxide $\left(\mathrm{HfO}_{2}\right)$ [7], zirconium oxide $\left(\mathrm{ZrO}_{2}\right)$ [8] and yttria stabilized zirconium oxide (YSZ) [9] are strongly recommended to replace $\mathrm{SiO}_{2}$. Among all, $\mathrm{CeO}_{2}$ is emerging as highly unique dielectric material due to its superior physical properties with chemical and thermal stability [10-14]. Typically, the $\mathrm{CeO}_{2}$ exhibits cubic structure having lattice parameter $a=0.541 \mathrm{~nm}$, which matches with silicon lattice parameter $a=0.543 \mathrm{~nm}$. This lattice matching property is highly interesting for epitaxial growth. These properties play an important role in the MIS, MIM and MOS capacitor applications [15-18] In addition, using $\mathrm{CeO}_{2}$ thin films wide variety of applications, which includes ultra-thin gate oxide in CMOS devices, capacitors, buffer layers, optical devices, electro-chromic devices, oxygen sensors, fuel cells and corrosion resistant coatings were proposed. The unique physical properties of $\mathrm{CeO}_{2}$, which include high dielectric constant, wide bandgap and low interface state density makes it as a potential candidate in gate dielectric applications [19-21].

$\mathrm{CeO}_{2}$ films are deposited using spray pyrolysis [25,26], sol-gel [27,28], thermal evaporation [29], electron-beam evaporation [30], RF magnetron sputtering [31] and pulsed laser deposition (PLD) techniques [11-14]. PLD is highly unique to produce high quality 
films in broad range of deposition conditions, such as substrate temperature, ambient gas, pressure of the gases, laser energy and repetition rate [32]. All these parameters can be effectively monitored to obtain the desired properties from thin films. In this research, we have used PLD technique to deposit $\mathrm{CeO}_{2}$ films on the Pt coated substrate $\mathrm{Pt}(111) / \mathrm{Ti} / \mathrm{SiO}_{2} / \mathrm{Si}$ (100), which is crucial for the electrical and dielectric property analysis. We have deposited $\mathrm{CeO}_{2}$ films at different temperatures to investigate the microstructural, optical and electrical quality of the films. We have used to take advantage of the deposited thin film to measure electrical and dielectric properties through metal-insulator-metal structure.

\section{Materials and Methods}

$\mathrm{CeO}_{2}$ powder with $99.99 \%$ purity was compacted using uni-axial press by applying 120 bar pressure and the pellet size was maintained at a specification of $25 \mathrm{~mm}$ dia $\mathrm{x} 4 \mathrm{~mm}$ thick. The compact green pellet was sintered at $1473 \mathrm{~K}$ for 6 hours to improve the packing density $\sim 90 \%$. Prior to deposition, the substrates were subjected to ultrasonic cleaning with acetone and dried under $\mathrm{N}_{2}$ gas flow. The experiments were carried out using $\mathrm{KrF}$ excimer laser $(\lambda \sim 248 \mathrm{~nm})$ and $300 \mathrm{~mJ}$ energy with $10 \mathrm{~Hz}$ repetition rate. The spacing between the substrate and target was kept at $4.5 \mathrm{~cm}$ with the optimized deposition time of 1 hour [11-14]. The films were prepared under $2 \times 10^{-2}$ mbar oxygen partial pressure and $300 \mathrm{~K}, 573 \mathrm{~K}$ and $873 \mathrm{~K}$ temperatures. Thickness of the films were measured from the height difference between coated and uncoated region. Dektak profilometer (DEKTAK 6M-stylus profiler Veeco, USA) was used for the thickness measurement. The crystallinity of the films were as analyzed using X-ray diffractometer (X'pert PW 3040 D-8, PANalytical) attached with point detector using $\mathrm{CuK} \alpha_{1}$ radiation. XRD pattern was recorded between $20-90^{\circ}$ and a step size of $0.2^{\circ}$ with a continuous scanning mode. The Scherrer formula is used to evaluate the crystalline sizes

$$
D=\frac{K \lambda}{\beta \operatorname{Cos} \theta}
$$


where $\beta=\sqrt{ }\left(B^{2}-b^{2}\right) K$ is Scherrer constant, $\lambda$ is the wavelength of incident radiation, , D-Crystallite size, $\beta$ is Full width at half maximum (FWHM), $\theta$-Angle of diffraction, BObserved FWHM of the film and b-Instrumental broadening. The photoluminescence spectra of the films were analysed using (Shimadzu, RF-5301PC) spectrofluorophotometer. The surface morphology and surface roughness of the films were analyzed using Atomic force microscope (AFM) (XE-100 Park systems) in contact mode. Dielectric properties of all films were measured using low frequency impedance analyser (4192A; HP, USA).

\section{Results and Discussion}

\subsection{Microstructural analysis}

The crystalline structure of the $\mathrm{CeO}_{2}$ films is investigated by XRD studies. Figure 1 shows the $\mathrm{XRD}$ pattern of the $\mathrm{CeO}_{2}$ thinfilms prepared at different temperatures. The $\mathrm{CeO}_{2}$ films exhibit cubic structure without any impurity phases. $\mathrm{CeO}_{2}$ film prepared at $300 \mathrm{~K}$ shows low intense peaks at angles of $28^{\circ}$ and $32^{\circ}$ corresponding to (111) and (200) reflections from the cubic $\mathrm{CeO}_{2}$ (JCPDS No. 34-0394). The films deposited at 573 and 873 K show the high intense peaks corresponding to (111) reflection, which indicated the improved crystallinity at higher temperature. Furthermore, the (111) reflection of the films deposited at 573 and $873 \mathrm{~K}$ temperatures is quite sharp with decreased FWHM as compared to $\mathrm{CeO}_{2}$ film deposited at $300 \mathrm{~K}$. The decrease of FWHM and increase of intensity revealed increase in crystallite size caused due to the ad-atom mobility, which in turn leads to the densification of $\mathrm{CeO}_{2}$ films. The calculated crystallite size is $19-22 \mathrm{~nm}$ range [11-13]. The high intense peaks at $40^{\circ}, 69^{\circ}$ and $86^{\circ}$ denotes the Pt peaks corresponding to (111), (220) and (222) respectively. Many researchers worked on $\mathrm{CeO}_{2}$ thinfilms for different applications. $\mathrm{CeO}_{2}$ films were deposited on $\mathrm{Si}(100)$ and glass substrates under various oxygen partial pressures and substrate temperatures using PLD [11,12] for optical applications. The results indicated the polycrystalline cubic nature of the films deposited at various oxygen partial 
pressures. $\mathrm{CeO}_{2}$ films deposited at various substrate temperatures (RT $-973 \mathrm{~K}$ ) and the XRD analysis indicated the polycrystalline cubic structure with (111) orientation at room temperature, while the orientation changed to (200) at higher temperatures [11]. Kanakaraju et al. [26] prepared the $\mathrm{CeO}_{2}$ films using ion beam sputtering and structural studies indicated (220) orientation up to $473 \mathrm{~K}$ and it changed to (200) orientation at $673 \mathrm{~K}$. Murugan et al deposited $\mathrm{CeO}_{2}$ films at $3 \times 10^{-4}$ mbar pressure using $\mathrm{RF}$ magnetron sputtering. XRD studies indicated cubic structure of the films [33].

AFM studies showed the images of the $\mathrm{CeO}_{2}$ films deposited at different temperature on $\mathrm{Pt}$ coated $\mathrm{Si}$ substrates. Fig. 2a shows the surface morphology of $\mathrm{CeO}_{2}$ film deposited at $300 \mathrm{~K}$ shows uniform distribution of vertically grown crystallites having dense microstructure over the entire substrate with good crystallinity. The $\mathrm{CeO}_{2}$ film prepared at $573 \mathrm{~K}$ (Fig. 2b) also shows similar surface morphology as observed in the film deposited at $300 \mathrm{~K}$. However, the crystallite size significantly increased for the $\mathrm{CeO}_{2}$ film prepared at 573 $\mathrm{K}$. On further increasing the deposition temperature to $873 \mathrm{~K}$ coalescence of crystallites is observed as shown Fig. 2c. The root mean square (RMS) surface roughness values were quantitatively measured using the AFM images and shown in Table 1. RMS surface roughness increased as $2.09 \mathrm{~nm}, 2.68 \mathrm{~nm}$ and $3.62 \mathrm{~nm}$ at 300,573 and $873 \mathrm{~K}$ temperatures respectively. The surface roughness increased due to increased crystallite size with the increasing substrate temperature. The increased crystallite size with increasing temperature is well correlated with the decrease of FWHM as observed in XRD study [11].

\subsection{Optical characterization}

Figure 3 shows the photoluminescence (PL) spectra of $\mathrm{CeO}_{2}$ thinfilms deposited at various temperatures. All thin films are excited at $325 \mathrm{~nm}$ wavelength using $\mathrm{He}-\mathrm{Cd}$ laser. PL spectra indicated a strong peak $\sim 366 \mathrm{~nm}$. The peak position is $\sim 366-368 \mathrm{~nm}$ wavelength, corresponding to the bandgap of $\sim 3.38-3.37 \mathrm{eV}$. The measured bandgap values well agreed 
with the bandgap of $\mathrm{CeO}_{2}$ reported in literature [12]. $\mathrm{CeO}_{2}$ film prepared at room temperature $(300 \mathrm{~K})$ shows higher intensity and PL intensity is decreased at higher temperatures $(573 \mathrm{~K}$ and $873 \mathrm{~K})[13,30]$. The PL studies revealed the energy gap of 3.05 $3.10 \mathrm{eV}$ range[13].

\subsection{Electrical and Dielectric properties}

MIM capacitor structure is formed and its electrical properties are studied. Platinum electrodes with $1.54 \times 10^{-4} \mathrm{~cm}^{2}$ area was deposited on the surfaces of the $\mathrm{CeO}_{2}$ films to form a capacitor structure. Figure 4 shows the schematic of the MIM structure. The variation of leakage current densities $(J)$ of $\mathrm{CeO}_{2}$ films with respect to electric fields $(E)$ are shown in Fig. 5 as a function of temperature. The measured leakage current densities were $2.40 \times 10^{-5} \mathrm{~A} / \mathrm{cm}^{2}$ $(300 \mathrm{~K}), 1.8 \times 10^{-6} \mathrm{~A} / \mathrm{cm}^{2}(573 \mathrm{~K})$ and $2.0 \times 10^{-7} \mathrm{~A} / \mathrm{cm}^{2}(873 \mathrm{~K})$, respectively at $100 \mathrm{KV} / \mathrm{cm}$.

The leakage current density of the $\mathrm{CeO}_{2}$ thinfilm deposited at $873 \mathrm{~K}$, indicates the minimum leakage current density and was two orders magnitude lower than the $\mathrm{CeO}_{2}$ film deposited at $300 \mathrm{~K}$. The high electrical leakage of the $\mathrm{CeO}_{2}$ thin film deposited at room temperature might be attributed to the poor crystallinity. The improved crystallinity with better microstructure caused low leakage current density of the $\mathrm{CeO}_{2}$ films prepared at 573 and $873 \mathrm{~K}$, respectively $[1,2,5,16]$. In dielectric thin films, the oxygen vacancies are act as mobile carriers for the large conduction. The dielectric thin films with large concentration of oxygen vacancies lead high electrical leakage current. The low leakage current density at 873 $\mathrm{K}$, indicating the reduced oxygen vacancies in the film.

Frequency dependent dielectric constant was measured by varying frequencies from $10^{2}$ to $10^{6} \mathrm{~Hz}$ (Fig.6.). The dielectric constant values (c) for the $\mathrm{CeO}_{2}$ films were $6(300 \mathrm{~K}), 8$ $(573 \mathrm{~K})$ and $10(873 \mathrm{~K})$, respectively at $1 \mathrm{kHz}$ frequency. The observed dielectric loss values were $1.13(300 \mathrm{~K}), 1.07(573 \mathrm{~K})$, and $1.03(873 \mathrm{~K})$, respectively (Fig.6) at $1 \mathrm{kHz}$. The 
discrepancies in the dielectric constants and dielectric loss in these films might be due to the ionic vacancies and microstructural features, such as crystallite size and porosity [7].

Jung-Ho Yoo et al. [4] prepared $\mathrm{CeO}_{2}$ films by reactive DC magnetron sputtering and found the polycrystalline cubic structure of the films and these studies indicted the maximum capacitance $\left(\mathrm{C}_{\max }\right)$ and reduced leakage current with increasing interfacial $\mathrm{SiO}$ layer thickness. $\mathrm{CeO}_{2}$ films were prepared on $\mathrm{Si}$ (100) substrates by PLD method and formed a MOS capacitor $\left(\mathrm{Pt} / \mathrm{Si} / \mathrm{CeO}_{2} / \mathrm{Pt}\right)$ and were found to have a dielectric constant of $23[16]$. $\mathrm{CeO}_{2}$ films were prepared on silicon at various temperatures using atomic layer deposition technique [20]. The as-deposited films were cubic structure for $423 \mathrm{~K}-623 \mathrm{~K}$ temperatures range. The permittivities were found to be 25-42 range at $1 \mathrm{MHz}$ [20]. The $\mathrm{CeO}_{2}$ films prepared on Si (100) using electron beam evaporation at $473 \mathrm{~K}$ to form a MIS configuration and the $C-V$ plots showed dielectric constant value of 3.4 [23]. Anil $\mathrm{G}$ Khairnar et al [33] prepared $\mathrm{CeO}_{2}$ films of thickness $5 \cdot 56 \mathrm{~nm}$ using sol-gel spin coating technique and the $\mathrm{C}-\mathrm{V}$ measurement indicated the dielectric constant of 18.92 at $1 \mathrm{MHz}$.

El-Nahass et al investigated the structural, electrical and dielectric behavior of bulk $\mathrm{CeO}_{2}$. XRD results revealed the cubic structure of $\mathrm{CeO}_{2}$ powder. The conductivity of the charge carriers was analyzed using electrical modulus formulism [36]. Takenori Yamamoto et al. [37] theoretically studied the dielectric properties of $\mathrm{CeO}_{2}$ using first-principles. The dielectric constant values of the films were found to increase with lattice expansion and decrease of oxygen vacancy. The dielectric constants values of $\mathrm{CeO}_{2}$ calculated from the first-principles are in agreement with our experimental results. These results indicated that the dielectric properties of the films with frequency are strongly dependent on preparation method, microstructure and crystallite size, controlled by the growth temperature. 


\section{Conclusions}

$\mathrm{CeO}_{2}$ thinfilms were prepared on $\mathrm{Pt}(111) / \mathrm{Ti} / \mathrm{SiO}_{2} / \mathrm{Si}$ (100) substrates at different temperatures using PLD. XRD studies revealed the formation of polycrystalline ceria of cubic structure with (111), (200) and (222) orientation. The AFM analysis confirmed the nanocrystalline nature of the films and improved crystallinity with increasing temperatures. RMS surface roughness of the films is $\sim 2.1-3.6 \mathrm{~nm}$. The PL studies indicated the bandgap of $\sim 3.38 \mathrm{eV}$. The dielectric constant of $\mathrm{CeO}_{2}$ films was found to increase with increasing substrate temperature, while the dielectric loss was found decreasing with increase of temperature. The low leakage current density of $2.0 \times 10^{-7} \mathrm{~A} / \mathrm{cm}^{2}$ was obtained at $100 \mathrm{KV}$ at the 873Ktemperature. This is due to the lattice expansion, improved crystalline nature and decrease of oxygen vacancy formation as the substrate temperature increased.

\section{Acknowledgement}

The author C.M. Raghavan thanks Aston Institute of Photonic Technologies (AIPT), Aston University, Birmingham, UK for the award of Marie Skłodowska-Curie Research Fellowship. 


\section{References}

1. D. Rathee, M. Kumar and S.K. Arya, Int. J. Comp. Appl. 8, 10-17 (2010).

2. S. Guha and V. Narayanan, Annu. Rev. Mater. Res. 39, 181-202 (2009)

3. A. Kingon, J.P. Maria, S.K. Streiffer, Nature 406, 1032-1038 (2000)

4. Jung-Ho Yoo, Seok-Woo Nam, Sung-Kwan Kang, Yun-Ha Jeong, Dae-Hong Ko, JaHum Ku, Hoo-Jeong Lee, Microelectronic Engineering 56, 187-190 (2001).

5. S.K. Sahoo, M. Mohapatra, A.K. Singh, and Shashi Anand, Mater. Manuf. Process, 25, 982-989 (2010).

6. L.V. Qipeng. Shaoqian Zhang. Songwen Deng. Yinsheng Xu. Gang Li. Qingwei Li. Yuqi Jin. Surf. Coat. Technol. 320, 190-195 (2017).

7. B.H. Lee, L. Kang, R. Nieh, W. J. Qi and C. J. Lee, Appl. Phys. Lett. 76, 1926-1928 (2000).

8. H. C. Zhong, G. Heuss, V. Misra, H. Luan, C. Lee and D. L. Kwong, Appl. Phys. Lett. 78, 1134-1136 (2001).

9. S.J. Wang, S.Y. Xu, L.P. You, S.L. Lim and C. K. Ong, Semicond.Sci. Technol. 13, 362-367 (2000).

10. H. J. Quah, W.F. Lim, K.Y. Cheong, Z. Hassan and Z. Lockman, J. Crystal Growth 326, 2-8 (2011).

11. G. Balakrishnan, S. Tripura Sundari, P. Kuppusami, P. Chandra Mohan, M.P. Srinivasan, E. Mohandas, V. Ganesan, D. Sastikumar, Thin Solid Films 519, 2520$2526(2011)$.

12. G. Balakrishnan, P. Kuppusami, T.N. Sairam, R. Thirumurugesan, E. Mohandas, D. Sastikumar, J. Nanosci. Nanotechnol., 9, 5421-5424 (2009).

13. G. Balakrishnan C.M. Raghavan C. Ghosh R. Divakar E. Mohandas Jung Il Song S.I. Bae, Tae Gyu Kim, Ceramics International 39, 8327-8333 (2013).

14. G. Balakrishnan, P. Sudhakara, Abdul Wasy, Ha Sun Ho, K. S Shin, J.I. Song, Thin Solid Films 546, 467-471 (2014).

15. Markku Leskela, Kaupo Kukli, Mikko Ritala, J. Alloys and Compd 418, 27-34 (2006). 
16. Jyrki Lappalainen, Darja Kek, Harry L. Tuller, J. Euro. Ceram Soc. 24, 1459-1462 (2004).

17. Floiran Fernandez-Gutierrez, O. Sanchez Garrido, M. Hernandez-velez, R. M. Bueno and J. M. Martinez-Duart, Solid-State Electronics 42, 925-930 (1998).

18. Khushabu S. Agrawal, Vilas S. Patil, Anil G. Khairnar, Ashok M. Mahajan, J. Mat. Sci: Mater. EI 28, 12503-12508 (2017).

19 Y. Nishioka, S. Kimura, H. Shinriki, K. Mukai, J. Electrochem. Soc. 134, 410-415 (1987).

20. Yu Chen, Yanxiang Zhang, Jeffrey Baker, Prasun Majumdar, Zhibin Yang, Minfang Han, and Fanglin Chen, ACS Appl. Mater. Interfaces, 6, 5130-5136 (2014).

Yukie Nishikawa, Noburu Fukushima, Naoki Yasuda, Kohei Nakayama and Sumio Ikegawa, Jap. J. Appl. Phys. 41, 2480-2483 (2002).

26. Bârcă, E.S.; Filipescu, M.; Luculescu, C.; Birjega, R.; Ion, V.; Dumitru, M.; Nistor, L.C.; Stanciu, G.; Abrudeanu, M.; Munteanu, C.; Dinescu, M. Appl. Surf. Sci. 363, 245-251 (2016).

27. S. Kanakaraju, S. Mohan, A.K. Sood, Thin Solid Films 305, 191-195 (1997).

28. Asmaa Eltayeb.; Rajani K. Vijayaraghavan.; Anthony P. McCoy.; Joseph Cullen.; Stephen Daniels.; Enda McGlynn. Thin Solid Films, 603, 363-370 (2016).

29. W. Ming, K.L. Choy, J. Cryst. Growth, 284, 464-469 (2005).

30. Agata Sawka, Andrzej Kwatera, Paweł Andreasik, Mater. Lett. 204, 39-41 (2017).

31. Tadeusz Wiktorczyk.; Piotr Biegan`ski.; Eunika Zielony. Opt. Mater. 34, 2101-2107 (2012).

32. F.C. Chiu, C.M. Lai, J. Appl. Phys. D: Appl. Phys. 43, 075104 (2010).

33. D.B. Chrisey, G.B. Hubler (Eds), Pulsed Laser Deposition of Thin Films, Wiley, New York, 1994.

34. R. Murugan, G. Vijayprasath, T. Mahalingam, Y. Hayakawa, G. Ravim. J. Mat. Sci: Mater. EI. 26, 2800-2809 (2015).

35. Anil G Khairnar and Ashok M Mahajan, Bull. Mater. Sci., 36, 259-263 (2013).

36. M.M. EI-Nahass, A.M. Hassanien, A.A. Atta, Emad M.A. Ahmed, Azad A. Ward, J. Mat. Sci: Mater. EI. 28, 1501-1507 (2017).

37. Takenori Yamamoto, T, Hiroyoshi Momida, Tomoyuki Hamada, Tsuyoshi Uda, Takahisa Ohno, Thin Solid Films 486, 136-140 (2005). 


\section{Figure captions}

Fig.1 XRD patterns of the $\mathrm{CeO}_{2}$ thin films deposited at 300,573 and $873 \mathrm{~K}$ on $\mathrm{Pt}$ $(111) / \mathrm{Ti} / \mathrm{SiO}_{2} / \mathrm{Si}(100)$ substrates.

Fig. 2 3D and 2D AFM images of $\mathrm{CeO}_{2}$ films deposited at different substrate temperature (a) $300 \mathrm{~K}$ (b) $573 \mathrm{~K}$ (c) $873 \mathrm{~K}$.

Fig. 3 PL spectra of the $\mathrm{CeO}_{2}$ films deposited at different substrate temperature.

Fig. 4 Schematic diagram of the deposited MIM Capacitor structure

Fig.5 Effect of applied electric field on Leakage current density of $\mathrm{CeO}_{2}$ films

Fig. 6 Frequency dependent dielectric properties of the $\mathrm{CeO}_{2}$ thin films deposited at different temperature. 


\section{Table captions}

Table 1 Crystallite size, RMS roughness and Dielectric constant of the $\mathrm{CeO}_{2}$ films deposited at different substrate temperature.

\begin{tabular}{|c|c|c|c|c|c|c|c|}
\hline S. & Substrate & Oxygen & Thickness & Crvstallite & RMS & Bandoan & Dielectric \\
\hline \multirow[t]{3}{*}{ No } & temperature & partial & $(\mathrm{nm})$ & size $(\mathrm{nm})$ & roughness & $(\mathrm{eV})$ & constant \\
\hline & & pressure & & & $(\mathrm{nm})$ & & \\
\hline & & (mbar) & & & & & \\
\hline 1 & $300 \mathrm{~K}$ & $2 \times 10^{-2}$ & 300 & - & 2.1 & 3.37 & 6 \\
\hline 2 & $573 \mathrm{~K}$ & $2 \times 10^{-2}$ & 300 & 19 & 2.7 & 3.38 & 8 \\
\hline 3 & $873 \mathrm{~K}$ & $2 \times 10^{-2}$ & 300 & 22 & 3.6 & 3.37 & 10 \\
\hline
\end{tabular}




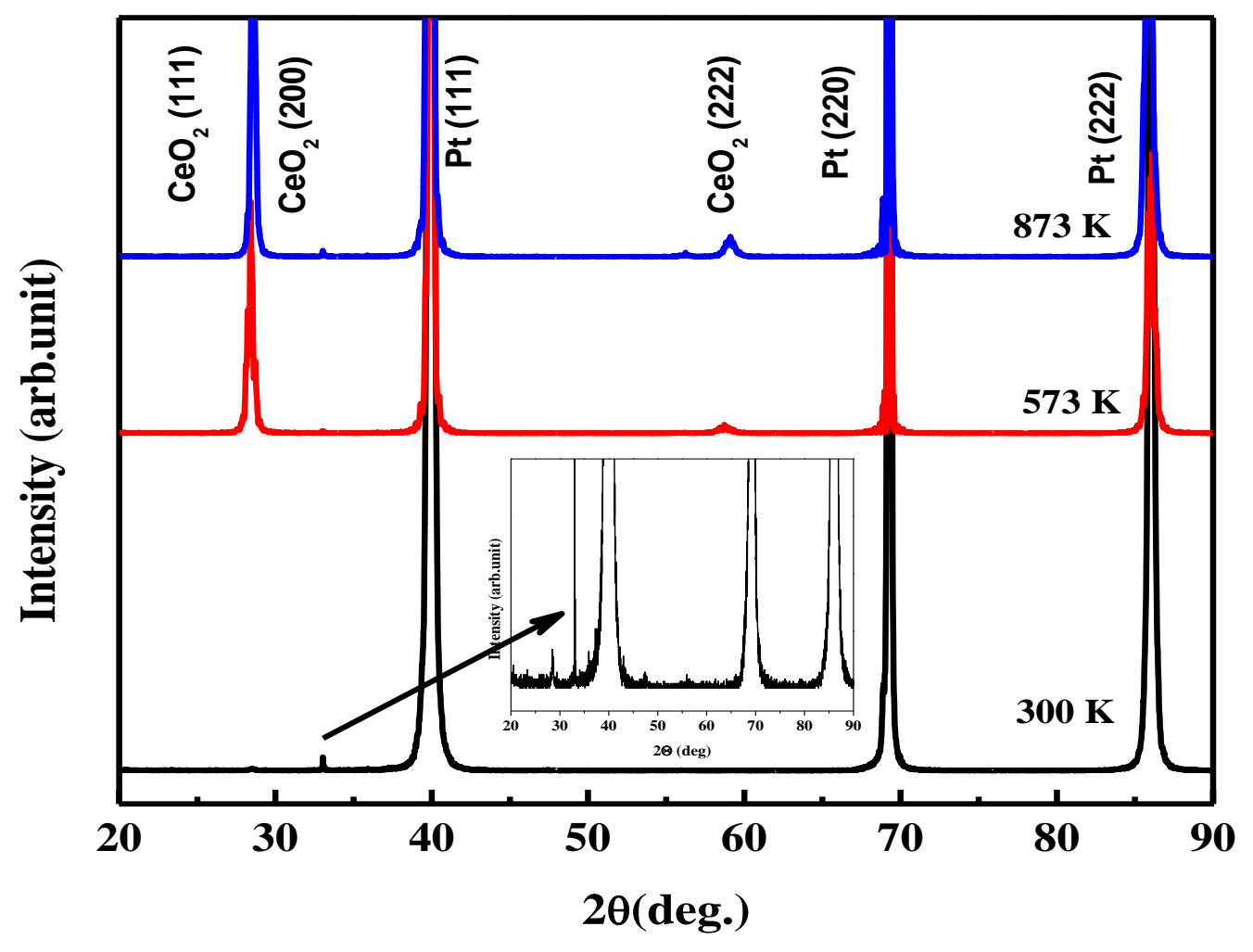

Fig.1 XRD pattern of the films deposited at various substrate temperatures 

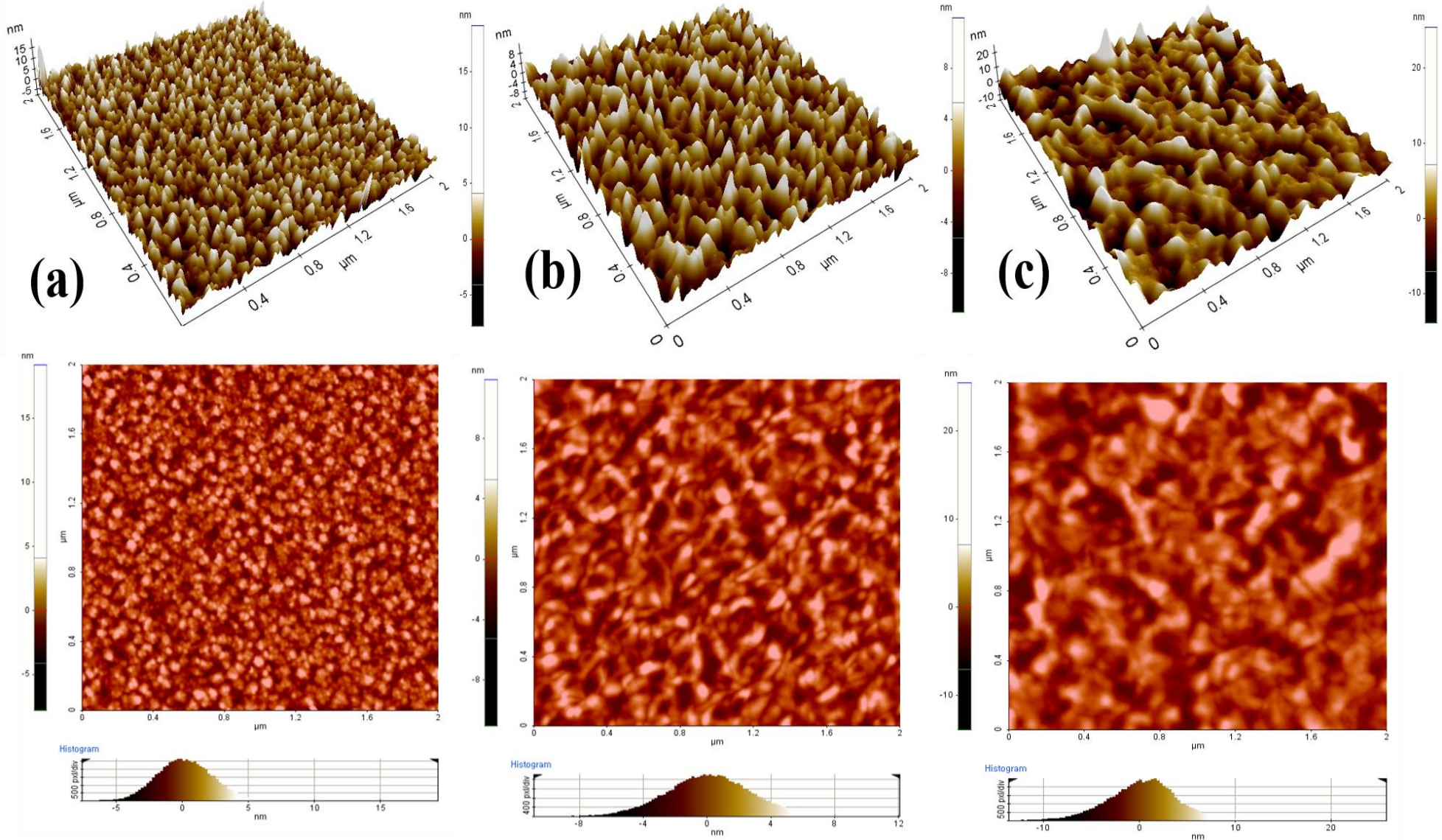

Fig.2 3D and 2D AFM images of $\mathrm{CeO}_{2}$ films deposited at different substrate temperature (a) $300 \mathrm{~K}$ (b) $573 \mathrm{~K}$ (c) $873 \mathrm{~K}$ 


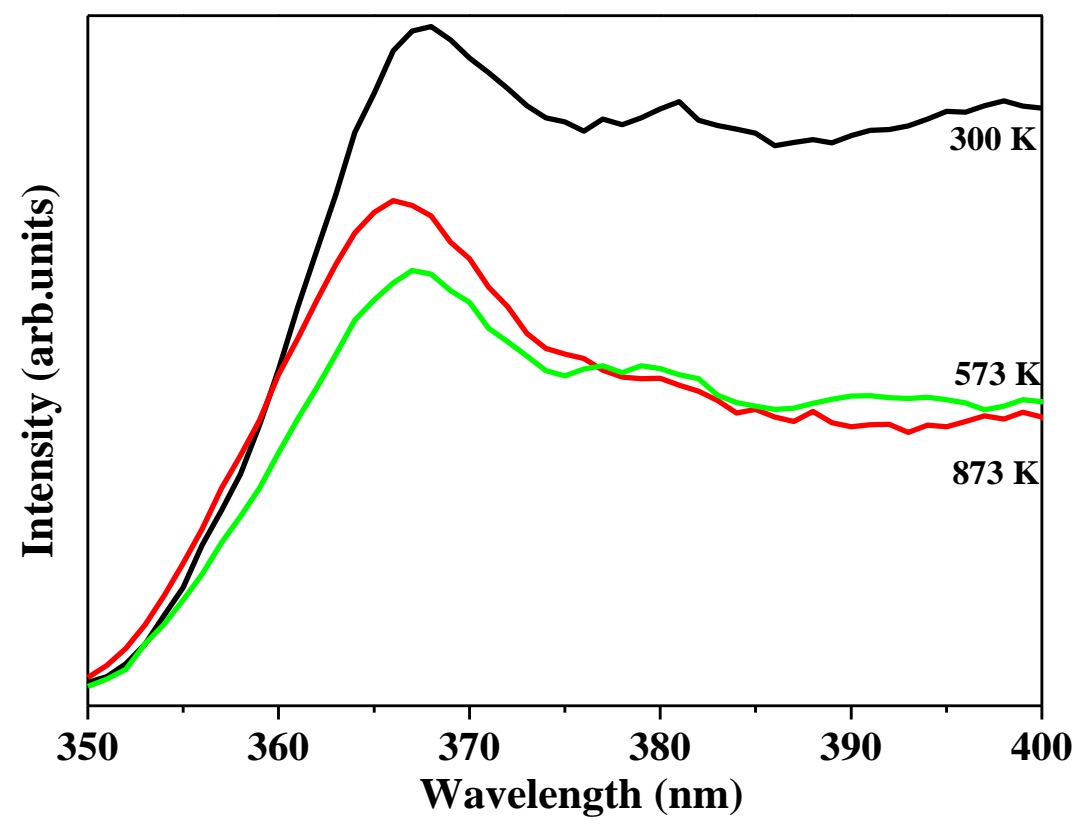

Fig.3 PL spectra of the $\mathrm{CeO}_{2}$ films deposited at different substrate temperature

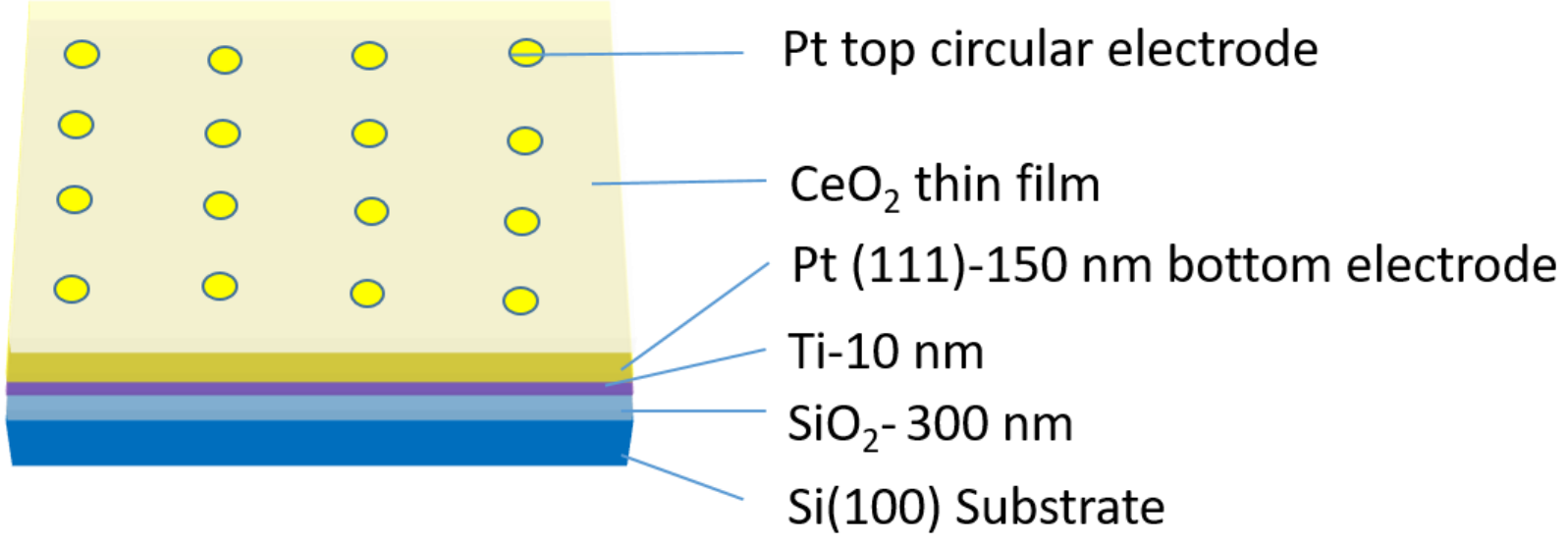

Fig.4 Schematic diagram of the device structure 


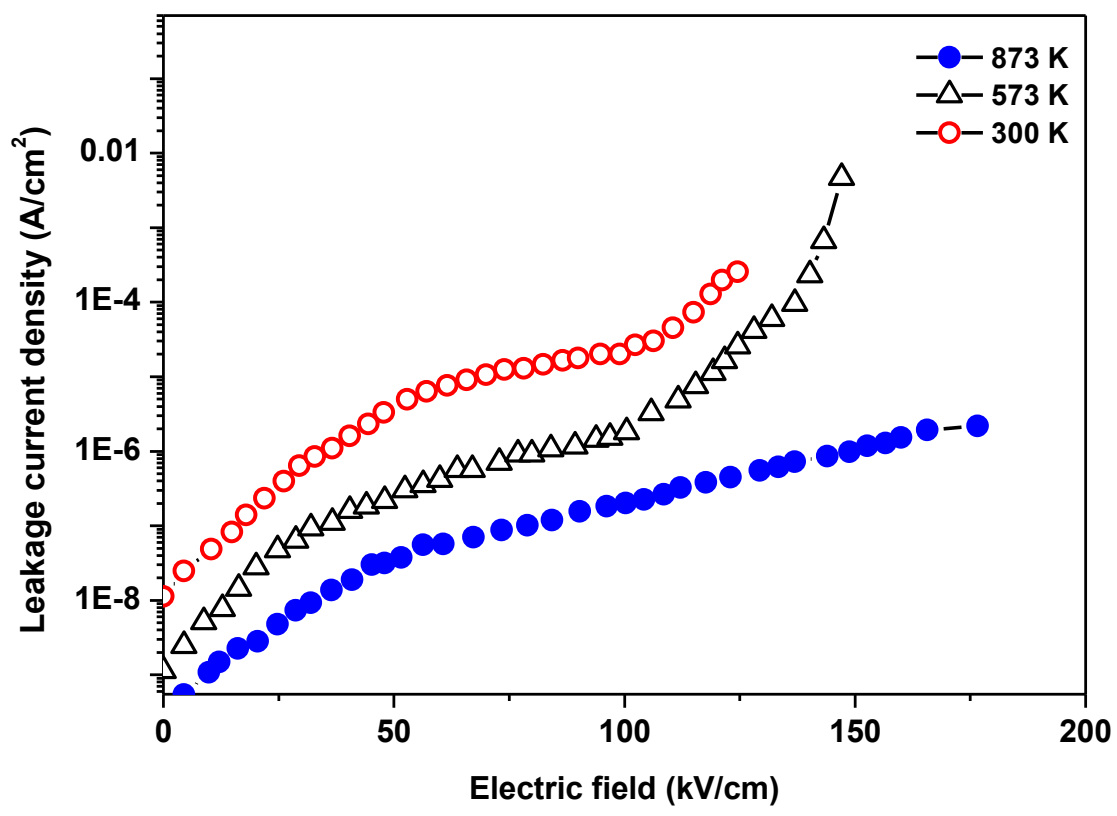

Fig.5 Effect of applied electric field on Leakage current density of $\mathrm{CeO}_{2}$ films

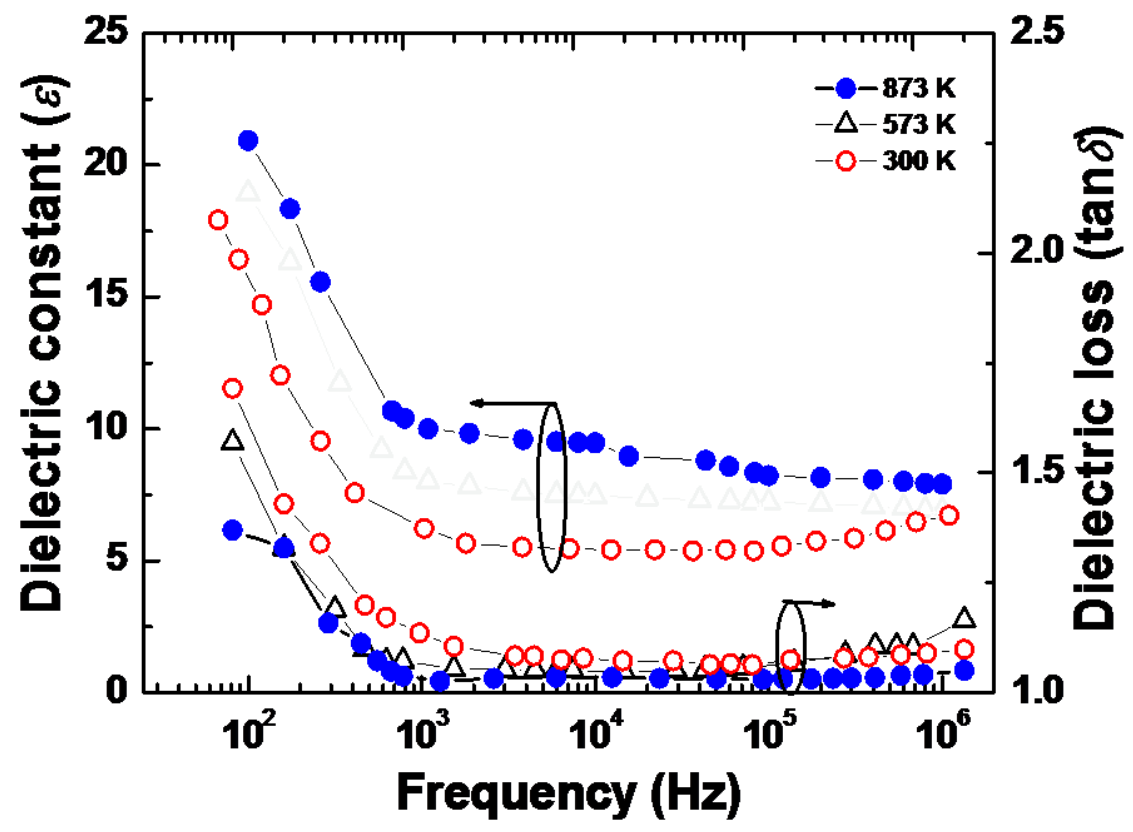

Fig.6 Frequency dependent dielectric properties of the $\mathrm{CeO}_{2}$ thin films deposited at different temperature. 